\title{
La economía política de la comunicación vista desde América Latina
}

\author{
Martín Becerra1 \\ Universidade Nacional de Quilmes - CONICET \\ Guillermo Mastrini 2 \\ Universidade de Buenos Aires
}

\begin{abstract}
Resumen: El presente artículo recupera el aporte del investigador argentino Heriberto Muraro, quien en los años ochenta trazó, a partir del examen crítico de las contribuciones de la economía política de la comunicación y el desarrollo de las industrias de la cultura como objeto de análisis, una propuesta de memorandum o agenda de prioridades para el campo de estudios en América Latina. A partir de los procesos de convergencia, globalización $y$ diseminación de las tecnologías de información y comunicación registrados en las últimas dos décadas y media, con la consecuente mutación de los procesos productivos del sector, se contrastan las investigaciones que se realizan en Iberoamérica con la agenda propuesta por Muraro, reformulándola y discutiendo algunos de sus supuestos. Asimismo, se propone una clasificación de las principales contribuciones en el marco de los estudios de economía política de la comunicación y la cultura en la región.
\end{abstract}

Palabras claves: Economía Política de la Comunicación; América Latina; Heriberto Muraro; Desarrollo de las Industrias de la Cultura.

\footnotetext{
1 Professor e pesquisador de la Universidade Nacional de Quilmes y del Consejo Nacional de Investigaciones Cientíticas y Tecnológicas (CONICET). Autor, em parceira com Guillermo Mastrini, de " Periodistas y magnates: estructura y concentración de las industrias culturales en América Latina" (Buenos Aires, editorial Prometeo, 2006) y autor de "Sociedad de la Información: proyecto, convergencia, divergencia" (Buenos Aires, editorial Norma, 2003).

2 Professor e pesquisador de la Universidade de Buenos Aires. Autor, em parceira com Martín Becerra, de " Periodistas y magnates: estructura y concentración de las industrias culturales en América Latina" (Buenos Aires, editorial Prometeo, 2006) e organizador da coletânea Mucho ruido, pocas leyes. Economía y políticas de comunicación en la Argentina (Buenos Aires: La crujía, 2005).
} 
Resumo: $O$ presente artigo recupera o aporte do pesquisador argentino Heriberto Muraro, que nos anos oitenta traçou, a partir de um exame crítico das contribuições da economia política da comunicação e do desenvolvimento das indústrias culturais como objeto de análise, uma proposta de memorandum ou agenda de prioridade para o campo de estudos na América Latina. A partir dos processos de convergência, globalização e disseminação das tecnologias da informação e da comunicação registrados nas duas últimas décadas e meia, com a conseqüente mutação dos processos produtivos do setor, contrastamos as investigações que se realizam na América Ibérica com a agenda proposta por Muraro, reformulando-a e discutindo alguns de seus pressupostos. Ao mesmo tempo, propomos uma classificação das principais contribuições no marco dos estudos de economia política da comunicação e da cultura na região.

Palavras-chave: Economia Política da Comunicação; América Latina; Heriberto Muraro; Desenvolvimento das Indústrias Culturais.

\section{Introducción}

Después de un período de reflujo y retroceso coincidente con la derrota representada por el archivo de las posiciones identificadas con el "Nuevo Orden Mundial de la Información y la Comunicación" (NOMIC), los estudios de economía y política de la comunicación han resurgido en América Latina en los últimos diez años. La necesidad de producir información, conocimientos, reflexiones y suscitar debates sobre la estructura y los movimientos del dinámico sector de la información, la comunicación y la cultura, la mencionada revitalización de los estudios de economía política de la comunicación merece ser analizada a partir de sus principales tendencias, sus aportes más destacados y, también, de sus "zonas erróneas". 
Para emprender dicho análisis, el presente artículo está organizado en base al texto de Heriberto Muraro, "Economía y Comunicación: convergencia histórica e inventario de ideas. Con especial referencia a América latina” (1987) que, elaborado inicialmente en 1984, proponía una agenda para los estudios afines al campo de la economía de la comunicación y la cultura.

Aunque parezca paradójico utilizar una referencia con más de dos décadas frente al propósito de someter a análisis el presente y la actualidad de los estudios de economía política de la comunicación, el texto de Muraro (como buena parte de la obra de su autor) postulaba un panorama original de organización de las contribuciones realizadas sobre la convergencia y los múltiples condicionamientos entre la comunicación y la economía fundamental --aunque no únicamente-- en América latina en los años posteriores a los debates enmarcados en el NOMIC. La resonancia de un presente cuya agenda de políticas comunicacionales se construye a modo de antítesis (es decir, como negación) de la agenda del NOMIC permite, al mismo tiempo, reconocer la pertinencia de su invocación para comprender claves centrales de la trama actual de la comunicación.

En aquel artículo, interesaba especialmente a Muraro definir las zonas vacantes y necesitadas de producción de conocimientos, lo cual reviste significación a la luz de la evolución los sectores industrializados de la cultura, la información y la comunicación en las dos décadas posteriores a la aparición del mencionado texto.

\section{El memorandum de Muraro}

En un esfuerzo por sistematizar a modo de memorandum los "espacios (en aquel entonces) poco explorados" que serán considerados en las páginas siguientes, Muraro identificaba la siguiente agenda:

1. El análisis de las industrias culturales en cuanto complejos integrados técnicamente y por reglas económicas que son específicas de este sector productivo. En este aspecto conviene enfatizar la necesidad de elaborar estudios sobre la producción cultural masiva que vayan más allá de los consabidos análisis de las relaciones de propiedad. 
2. El examen de las correlaciones y mutuas determinaciones existentes entre los procesos macroeconómicos y los comunicacionales; en especial, todo lo referido a la socialización de los agentes económicos.

3. La incidencia de las nuevas tecnologías en la organización técnica, financiera o administrativa de las actividades económicas.

4. Con mayor generalidad: el papel de la transmisión de información y otras actividades comunicativas en la organización cotidiana de las actividades económicas tales como la determinación de precios a corto plazo, la evolución de los mercados de valores, o bien el funcionamiento de los mercados laborales.

5. El papel de los medios masivos, o de circulación restringida, en la toma de decisiones de los agentes económicos ante políticas gubernamentales de promoción del desarrollo o de control económico de la coyuntura.

6. En último término: la elaboración de un modelo más amplio de la acción racional dentro del cual tengan cabida los procesos de transmisión de información y comunicación (Muraro, 1987).

Después de enunciar este sintético memorandum, el autor exponía en su texto, con mayor profundidad analítica, las principales corrientes de pensamiento sobre desarrollo económico y comunicación social, asumiendo la centralidad de la mass communications research, así como sus correspondientes respuestas históricas, algunas de ellas generadas en América latina (teoría de la dependencia, interpretaciones del subdesarrollo). Asimismo, la estructura del texto presentaba la crítica de la teoría de la dependencia en el campo comunicacional y los desafíos implícitos en la revolución "comunicacional y económica" implícita en el salto convergente de las nuevas tecnologías, para concluir con la (re)configuración de flujos de información "transnacional". Cabe recordar que el propio Muraro había realizado muy tempranamente una acertada crítica sobre algunos problemas que presentaban algunas lecturas de la teoría de dependencia para un estudio adecuado de la estructura económica de las industrias culturales (1974). 
No es propósito del presente artículo reseñar la exposición de Muraro sobre las líneas fundantes de los estudios de economía y política de la comunicación, sino dialogar críticamente con la síntesis a la que el autor arriba:

\section{Debatiendo el memorandum}

Muraro presentaba el memorandum de seis puntos cuya recuperación crítica se revela pertinente en función de la actualidad. Pero dado que este "presente" se ha ido gestando como producto de procesos y movimientos que excedían la posibilidad de prospectiva de principios de los ochenta (puesto que aquel momento histórico contaba con determinaciones inmediatas fruto de otros procesos y movimientos de las que el analista no podía prescindir) es intención de las páginas que siguen elaborar críticamente la síntesis del autor que tomamos como referencia.

En términos contextuales, conviene señalar en primera instancia que si Muraro concentraba su mirada en América latina, resulta necesario ampliar hoy el memorandum a Iberoamérica, dado que se trata de un espacio que cuenta con campos de colaboración intelectual, redes de cooperación científica e itinerarios de producción de investigación que dan cuenta de una configuración que, sin llegar a ser un mismo y homogéneo espacio (ni tampoco necesariamente "horizontal" o exento de condicionamientos de agenda y de campos), presenta agendas relativamente concurrentes. Esta situación se corresponde plenamente con procesos económicos, que tienen su correlato en el sector de la comunicación y la cultura, con lógicas de desarrollo marco complementarias (lo que no significa simétricas ni equivalentes), lo cual por último redunda en la presencia de capitales dominantes en el espacio iberoamericano con especial incidencia en la producción, tratamiento y distribución de bienes y servicios de la comunicación y la cultura.

En consecuencia, en virtud de los cambios estructurales acaecidos en las dos últimas décadas y de las contribuciones crecientemente realizadas por estudios de economía política de la comunicación, es posible abordar una reelaboración de los seis puntos del memorandum propuesto por Muraro en los siguientes términos: 
1. Las correlaciones y mutuas determinaciones existentes entre los procesos macroeconómicos y los comunicacionales. Los medios (masivos o de "nicho"), la socialización y el comportamiento de los agentes económicos. La información y su influencia en el entramado económico-financiero.

2. La incidencia de las nuevas tecnologías en la organización técnica, productiva, financiera o administrativa de las actividades económicas (incluyendo especialmente la cuestión laboral).

3. La incorporación de dinámicas socioeconómicas a lo cultural, así como los condicionamientos socioculturales de lo económico.

4. Las industrias culturales (en tanto que complejos económicos y tecnológicos integrados) más allá de los análisis de las relaciones de propiedad.

5. Política y legislación sobre industrias culturales en el contexto de la convergencia tecnológica y la concentración económica.

Esta agenda se propone en función de una necesaria actualización del trabajo de Muraro realizada a partir de las transformaciones acaecidas en el sector de la industrias culturales, manteniendo a la vez el objetivo de retomar y problematizar los trabajos fundantes de la economía política de la comunicación. Esta labor, es importante señalarlo, en ocasiones es realizada en términos asimétricos en un campo (el comunicacional) que se estructura con la dominancia de una mirada poco afecta a la consideración analítica de procesos económicos e histórico-políticos y, en última instancia, renuente a la elucidación de la complejidad en la que las industrias culturales traman una estructura de relaciones de poder.

A continuación se presentan a modo de guía algunas de las referencias más relevantes de la actual producción iberoamericana que atiende a los cinco puntos con los que se propone reformular la agenda iniciada por Muraro en su artículo:

1. Correlaciones y mutuas determinaciones entre procesos macroeconómicos y comunicacionales. Medios (masivos o de "nicho"), socialización y 
comportamiento de los agentes económicos. La información y su influencia en el entramado económico-financiero.

Uno de las grandes transformaciones de la economía mundial en las últimas décadas fue sin duda el creciente peso económico de las industrias culturales en todos los indicadores económicos. Este fenómeno permite afirmar que el sector cultural ha alcanzado una definitiva madurez económica y una plena mercantilización. Es claro que aún subsisten áreas de la producción cultural no vinculadas a la industria cultural, pero estas son marginales en términos de consumo popular.

En términos generales el proceso de globalización es parte constitutiva de la nueva dinámica de las industrias culturales a la vez que no hubiera sido posible sin una efectiva participación de las nuevas tecnologías de información y comunicación. Alain Herscovici (1994) ha estudiado cómo se vinculan los efectos de la desterritorialización económica con la producción cultural. Por su parte un excelente volumen de recopilación de José Vidal Beneyto (2002) da cuenta de los principales desafíos que la globalización ha abierto en la definición de un nuevo concepto de esfera pública y de regulación del sistema de medios. Si bien no todos los autores comparten un enfoque basado en la economía política, los trabajos de Armand Mattelart, Enrique Bustamante o Robert McChesney resultan esclarecedores en este sentido.

Una de las carencias que se observan para el enfoque de la economía política es analizar el impacto del llamado "gobierno global de la comunicación". Dado que la producción cultural es considerada una mercancía más para muchos gobiernos y organismos internacionales como la Organización Mundial de Comercio, la regulación de su producción e intercambio ya forma parte de las agendas internacionales que intervienen en el tema. Si bien varios trabajos han mostrado preocupación por el tema, pocos muestran por ahora estudios empíricos que permitan avanzar analíticamente sobre sus consecuencias.

Más relevados han sido los procesos de integración regional y sus impactos sobre el sector audiovisual y las telecomunicaciones. En este sentido se destacan los trabajos producidos por el "Proyecto Monarca" que analizó el impacto del NAFTA en los países 
del norte del continente (Crovi, 1995) También sus trabajos quedaron reflejados en una publicación colectiva dirigida por Guillermo Mastrini y César Bolaño (1999) con destacados aportes de Gaëtan Tremblay y Delia Crovi. Más concentrado en el sector audiovisual y especialmente en el cinematográfico, Enrique Sánchez Ruiz ha realizado aportes importantes. En todos los casos se destacan los enormes desafíos que la liberalización del comercio implica para el sostenimiento de las políticas culturales destinadas a fomentar la producción local.

Es probable que debido al menor nivel de acuerdo y desarrollo del Mercosur, los trabajos sobre integración y cultura en la región no hayan alcanzado todavía un desarrollo significativo. Sin embargo, es de destacar los aportes de los trabajos colectivos coordinados por Sergio Cappareli (1999) y Luis Albornoz (2000) donde aparecen una serie de estudios que permiten comparar el impacto del período neoliberal sobre la estructura de las industrias culturales del bloque. Asimismo, Othon Jambeiro (2000) realizó un análisis comparativo de la regulación existente en materia televisiva en el Mercosur, mientras que Octavio Getino (2000) examinó la estructura económica de las industrias culturales en dicho bloque regional.

Si bien parten de una perspectiva que no está directamente vinculada a la economía política, los trabajos reunidos por Néstor García Canclini y Carlos Monteta en su libro Las industrias culturales en la integración latinoamericana (1999) complementan los estudios citados, y en los casos particulares Germán Rey y Rafael Roncagliolo, presentan una importante contribución para comprender los procesos de socialización a partir de las industrias culturales.

La gran mayoría de los estudios mencionados en este apartado reconocen la influencia del trabajo de Ramón Zallo (1988) que estableció las bases para el estudio de las industrias culturales desde la perspectiva de la economía política de la región. En América Latina resulta de especial interés la producción de Cesár Bolaño (2000) que revisa los principales postulados de la economía política y realiza un aporte original, y la de Alain Herscovici, quien retoma las premisas de la escuela francesa de la economía de la cultura. 
2. Incidencia de las nuevas tecnologías en la organización técnica, productiva, financiera o administrativa de las actividades económicas (incluyendo especialmente la cuestión laboral).

La centralidad económica de los procesos y actividades culturales, comunicacionales e informacionales convocan crecientemente a investigadores iberoamericanos a analizar tanto desde el plano conceptual sus lógicas de funcionamiento como generando estudios de carácter empírico. Referentes como Ramón Zallo (1988, 1992, 1995, 2000), César Bolaño (2000, Mastrini y Bolaño, 1999, Bolaño y Herscovici, 2004), Heriberto Muraro (1987), Claudio Katz (1997, 1998, 2001), Guillermo Sunkel (1999, 2001), Alain Herscovici (1994, 2004), Luis Stolovich (1997, 2001), Delia Crovi Druetta (2001, 2004) o Manuel Castells $(1994,1995)$ ponen el acento en el cambio de modo de desarrollo, que algunos de ellos nombran como modo de acción, proceso que independientemente de los términos, es protagonizado por las actividades infocomunicacionales. El influjo directo de estas actividades en el conjunto de los procesos productivos (sean o no comunicacionales) es el disparador del interés de muchos de los autores mencionados.

La transformación de los procesos y circuitos productivos, las rutinas laborales afectadas por la diseminación urbi et orbe de las tecnologías convergentes que permiten procesar y comunicar volúmenes de información inimaginados hace sólo cuarenta años (en este sentido destacamos el trabajo de los colegas Postolski, Santucho y Rodríguez, 2004), es entonces uno de sus principales temas, tal como se sostiene en el libro Sociedad de la Información: proyecto, convergencia, divergencia (Becerra, 2003). La naturaleza intangible de muchos de los intercambios económicos (básicamente los financieros) que afectan la estructura productiva y la performance de países y regiones enteras del planeta se retroalimenta con las tendencias globalizadoras del capital que también atienden, como objeto crítico de análisis, algunos de los autores de esta línea de aproximación a la economía política de la comunicación.

La doble faceta de la comunicación, que como recurso es infinito pero que sin embargo responde crecientemente a una lógica de intervenciones económicas, lógica que 
paradójicamente suele expandirse bajo la coartada de los bienes escasos, se perfila como un instrumento ideal para abordar el análisis de los cambios sociales en curso. Más aún cuando "el carácter mercantil que adquiere el uso de los recursos informacionales implica su sometimiento inevitable a las leyes que gobiernan la producción y realización general de mercancías" (Torres López y Zallo, 1991: 64).

3. Incorporación de dinámicas socioeconómicas a lo cultural, así como los condicionamientos socioculturales de lo económico.

Escribe Néstor García Canclini: "las teorías culturales y artísticas (Bourdieu, Eco) demostraron largamente que la creación cultural se forma también en la circulación y recepción de los productos simbólicos. Es necesario, entonces, dar importancia en las políticas culturales a esos momentos posteriores a la generación de bienes y mensajes, o sea al consumo y apropiación de las artes y los medios masivos" (2004).

Los conceptos de hibridación y ecualización como funciones típicas de la intervención de las industrias de la cultura en la configuración de sociedades heterogéneas y contradictorias a los que se alude frecuentemente al referir la obra de García Canclini como también las contribuciones de George Yúdice (2002, 2003), permiten dar cuentas de esta tercera línea de trabajo. Línea que se aparta de los objetos y de las metodologías de estudio prototipos de la economía política de la comunicación, sin embargo los autores mencionados, así como Omar Rincón o Germán Rey realizan contribuciones insoslayables a la hora de comprender las tensiones (marcadamente políticas) que se instituyen entre la estructura económica y las dinámicas socioculturales.

Puede ensayarse la hipótesis de que esta línea de exploración permite construir articulaciones entre los aportes de los estudios de la cultura y los de la economía política en un mundo cuyos trazos de globalización son ineludibles para abordar las rutinas culturales de los diferentes grupos sociales, en las distintas latitudes. En palabras de Yúdice, "la noción de cultura como recurso implica su gestión, un enfoque que no era característico de la alta cultura ni de la cultura cotidiana, entendida en un sentido antropológico. Y para 
complicar aún más las cosas, la cultura como recurso circula globalmente, con creciente velocidad" (2002: 16).

La ciudadanía como carta de presentación con autonomía relativa frente a los poderes establecidos no puede sostenerse, según esta línea de pensamiento, sino entendiendo sus lazos constituyentes con las prácticas de consumo y de interacción económica en un contexto de oligopolización y de agregación de valor cultural de los intercambios en la economía. La noción de ciudadanía no puede escapar a esa traza doblemente significativa: cultural y económica. Autores como Renato Ortiz, de Octavio Ianni, Rosana Reguillo, Aníbal Ford o José Carlos Lozano Rendón realizan aportes sustanciales al análisis de la producción cultural y sus peculiaridades colaborativas.

4. Industrias culturales (en tanto que complejos económicos y tecnológicos integrados) más allá de los análisis de las relaciones de propiedad.

El estudio de las industrias culturales en América latina, que tuvo sus inicios en el trabajo señero de Antonio Pasquali en la década del 60, cuenta con una vasta tradición. Si como señalara oportunamente Muraro durante gran parte de la década del 70 existió una fuerte tendencia a realizar una aproximación instrumental a la economía de las industrias culturales con el objetivo de analizar la matriz ideológica de los mensajes televisivos a través del análisis de propiedad, a partir de los 90 aparece y se consolida una corriente de estudios que busca comprender las dinámicas económicas involucradas en la producción cultural. Por supuesto que esto no supone abandonar el estudio de las relaciones de poder vinculadas a la producción cultural, pero ésta deja de estar subordinada a ser meramente un factor de reproducción ideológica.

En este punto es posible distinguir estudios teóricos, de tipo empírico y/o de análisis "de caso", que ilustran sobre las principales coordenadas de funcionamiento de las industrias culturales no sólo tomando en consideración su estructura de propiedad y las mutaciones de escala registradas en los últimos quince años a partir de los procesos de transferencia de activos estatales al capital privado, sino también las rutinas productivas de 
estas industrias, sus políticas de programación y estrategias de consolidación de posiciones dominantes en mercados de accesos restringidos.

Como se ha señalado, el trabajo de Ramón Zallo (1988) constituye un punto de partida insoslayable para la revitalización de los estudios de economía política de la comunicación en el área latina. Su libro resulta un claro aporte para comprender la dinámica macroeconómica de las industrias culturales a través de un recorrido que reconstruye de las principales ramas de la producción: edición discontinua, edición continua y el audiovisual continuo.

Otro aporte teórico de importancia fue el que realizó Enrique Bustamante (1999) al analizar la estructura económica de la televisión a partir de las transformaciones propias del período neoliberal. Aún cuando no puede eludir el contexto europeo de transición hacia una televisión competitiva, el trabajo de Bustamante constituye un referente para el estudio de la televisión en América latina. Más recientemente, Bustamante (2003) dirigió un pormenorizado estudio de la influencia de los procesos de digitalización sobre las industrias culturales, con un enfoque abarcador que incluye los fenómenos de la convergencia, la concentración, las políticas culturales y los derechos de propiedad intelectual.

El tema - problema de la concentración también detenta una larga tradición en la región. Sin embargo, recién a comienzos de la década del 90 un estudio sistematizó el análisis macro y mezo económico de los grupos económicos, así como sus estrategias (Miguel, 1993). Juan Carlos Miguel completó en los inicios del siglo XXI su estudio con el análisis del vínculo entre concentración de la propiedad y la convergencia de los sectores del audiovisual y las telecomunicaciones (2003, Tremblay)

Con una propuesta vinculada al relevamiento empírico de los niveles de concentración, los autores del presente artículo hemos dirigido una investigación sobre estructura y niveles de concentración de las industrias culturales en América Latina. En este caso se trata de consolidar información básica para poder en el futuro vincular la estructura de la propiedad al problema del pluralismo informativo (Mastrini y Becerra, 2006). En el primer informe comparativo hemos podido constatar el altísimo nivel de concentración de las industrias culturales en América Latina. 
Estudios sobre industrias culturales, a modo de bileras productivas en el marco de las transformaciones convergentes, permiten a la vez comprender el funcionamiento de sectores clave de la cultura en plena transformación. Tales son los análisis de Juan Calvi, Gustavo Buquet y Luis Albornoz, entre otros.

Crecientemente se registran aportes realizados a partir del estudio de la estructura comunicacional en diversos países. En este sentido cabe mencionar los equipos de investigación dirigidos por José Carlos Lozano Rendón (Instituto Tecnológico de Monterrey) y Enrique Sánchez Ruiz (Universidad de Guadalajara) en México y los estudios de caso de Jenny Ampuero y Rosmery Machicado en Bolivia.

En Chile Guillermo Sunkel aplicó una mirada de mirada de la economía política de la comunicación al fenómeno de la concentración de la propiedad de los medios (SunkelGeoffroy, 2001). Por su parte, en Venezuela se destacan diversos trabajos de Carlos Guzmán Cárdenas $(2003,2004)$ sobre la dimensión económica de las industrias culturales en dicho país. También se encuentra una mirada renovada de la relación entre economía e industrias culturales en los trabajos del recientemente fallecido Luis Stolovich (2002, 2004) sobre las políticas audiovisuales y la estructura económica de la cultura en Uruguay.

En la Argentina, además de los aportes de Octavio Getino sobre la dimensión económica de las industrias culturales (1995) es importante destacar las contribuciones realizadas por el Grupo de Investigación en Economía política de la comunicación de la Universidad de Buenos Aires (Albornoz, Hernández, Mastrini, Postolski).

Finalmente en el caso brasileño hay que reseñar una vez más los aportes del texto compilado por Valerio Cruz Brittos y César Bolaño (2005) sobre el grupo Globo y los de Othon Jambeiro, especialmente referidos a la televisión.

5. Política y legislación sobre industrias culturales: el desafío del pluralismo y la diversidad en el contexto de la convergencia tecnológica y la concentración económica. 
Otro lugar donde los estudios de economía política de la comunicación han recuperado un importante nivel de intervención es en el terreno de las políticas de comunicación. No podía ser de otra manera, dado que los cambios en la legislación de las industrias culturales han acompañado su vertiginosa transformación económica. En este sentido cabe destacar que los nuevos estudios en la materia han superado algunos determinismos propios de algunos trabajos de la década del 70, desarrollando el problema en forma más compleja y más articulada con otras problemáticas.

En España sobresalen los trabajos del Instituto de la Comunicación de la Universidad Autónoma de Barcelona. El equipo que dirige Miquel de Moragas (María Isabel Fernández, Merce Díez, entre otros) desarrollan análisis originales sobre estructura, dinámica y financiamiento de las actividades audiovisuales y de prensa escrita, en colaboración con Emili Prado y Rosa Franquet. Cabe destacar, además de la importante labor producida desde el "Observatorio de políticas de comunicación" del InCom, el monográfico producido con motivo del XXV aniversario del informe MacBride, producido conjuntamente con el Consejo Audiovisual de Cataluña. El aporte original de Carles Llorens y su tesis sobre pluralismo, aunque esta se distancie de alguno de los postulados clásicos de la economía política, resalta en el marco teórico de los sólidos aportes del grupo dirigido por Alfonso Sánchez Tabernero. Un antecedente en materia de investigación en políticas de comunicación, vinculado en este caso a la estructura internacional se encuentra en el libro de Marcial Murciano (1992).

Con base en la Universidad Complutense de Madrid, aparecen los trabajos de Fernando Quirós Fernández y Ana Segovia, que han contribuido a profundizar el conocimiento sobre las políticas que derivaron en el fracaso de la etapa democratizadora previa al neoliberalismo. El trabajo de Segovia resulta un aporte para un mejor conocimiento de la escuela crítica norteamericana en lengua castellana. En el País Vasco, los últimos trabajos de Zallo y los de Patxi Azpillaga han mostrado una constante preocupación por el desarrollo de políticas culturales en las regiones. Otros investigadores que presentaron una temprana preocupación por las políticas en el sector audiovisual son Eduardo Giordano y Carlos Zeller (1999). En la misma línea se sitúan las contribuciones de la Universidad de Sevilla que dirige Francisco Sierra Caballero. 
Si en América Latina existió un decidido impulso para la formalización del concepto de políticas nacionales de comunicación en la década del 70, este tema no podía quedar relegado en la actualidad.

En Brasil existe un importante equipo de investigación liderado por el profesor Murilo César Ramos en la Universidad de Brasilia, que ha producido importantes aportes sobre las nuevas formas de regulación en materia audiovisual y de telecomunicaciones. Otras investigaciones importantes son la de Anita Simis vinculada a la industria del cine y la de Valerio Brittos en materia de televisión. Por su parte José Marques de Melo (2005) ha compilado un volumen en el que se recuperan los debates sobre el MacBride a épocas de la sociedad de la información.

En Argentina, hemos contribuido a reconstruir la historia de las políticas de comunicación en el país, tarea que se había postergado incluso en las décadas más fecundas (Mastrini, 2005). Asimismo, se han producido trabajos conjuntos de análisis de las políticas de comunicación en el Mercosur (Cappareli, 1999).

Otros aportes interesantes, con miradas más globales los constituyen los trabajos de John Sinclair (1999) sobre la televisión latinoamericana y de Elizabeth Fox y Silvio Waisbord (2002) que sirve para presentar un cuadro comparativo de la situación de los principales países de la región en materia de políticas de comunicación. En la mayoría de los casos los autores previenen sobre la falta de mecanismos democráticos en la definición de las iniciativas en el área.

Si bien no adscriben a una línea de trabajo vinculada a la economía política de la comunicación hay que destacar los trabajos de Valerio Fuenzalida (2000) y Omar Rincón (2001) en los que se actualiza el diagnóstico sobre la televisión pública en el continente. A su tradicional falta de independencia del poder gubernamental, la irrupción del neoliberalismo parece haber dejado a las emisoras de propiedad estatal con bajos índices de legitimidad que llevan a los autores a realizar un riguroso planteo sobre las modalidades de su reforma, tomando en consideración la dimensión del entretenimiento como esencia de las culturas populares, esencia que ha sido históricamente subestimada por muchos investigadores del campo de la economía política. 
En términos generales, todos los trabajos coinciden en destacar el profundo impacto que la ejecución de políticas de orientación neoliberal ha tenido sobre la estructura de las industrias culturales. En este sentido, se remarca que la convergencia tecnológica y la concentración económica constituyen un renovado desafío para la imprescindible democratización de la comunicación.

\section{Palabras finales}

Del repaso general de los trabajos producidos desde los estudios de economía política de la comunicación en Iberoamérica a partir de la reformulación de la agenda que de modo pionero y con el formato de memorandum presentaba hace más de dos décadas Heriberto Muraro, podemos concluir que se ha producido un importante avance en materia de producción de conocimiento de la relación entre economía y comunicación. Entendemos que en primer lugar se ha superado la aproximación instrumental que realizaron los estudios de comunicación a la estructura económica de las industrias culturales con el objetivo de inferir la intencionalidad de los mensajes. Por otra parte los estudios de economía política de la comunicación presentan una creciente amplitud que abarca el tradicional estudio de la estructura de las industrias culturales, las transformaciones en el sistema productivo y las políticas de medios, pero que también busca indagar en la incidencia de los desarrollos tecnológicos y en cómo las tendencias generales de la economía impacta específicamente en el área de la cultura.

De todas formas, en relación a los puntos presentados por Muraro, es preciso señalar que todavía son escasos los trabajos referidos a los problemas más específicos de la economía de la comunicación, sobre todo los referidos a la socialización de los agentes económicos, a las modalidades cambiantes de producción, diseminación y apropiación de tecnologías convergentes, a la incidencia de dichos cambios en la agenda gubernamental y en el comportamiento estratégico de las industrias culturales y a la producción de saberes a partir de la realización de estudios de tipo empírico y comparativo. Creemos que esta zona de vacancia se puede a la vez enunciar como un atractivo desafío para la comunidad de investigadores del campo. 


\section{Referencias:}

Albornoz, Luis (2005). Los diarios online de información general. El caso de los grandes periódicos en español. Madrid, España. Tesis de doctorado defendida en mayo de 2005 en la Facultad de Ciencias de la Información de la Universidad Complutense de Madrid.

Albornoz, Luis (2000) (Coord.), Al fin solos... La nueva televisión del MERCOSUR, Ediciones Ciccus-La crujía, Buenos Aires, 290p.

Becerra, Martín (2005) "Las políticas de infocomunicación ante la Cumbre Mundial de la Sociedad de la Información”, en Quaderns del CAC n²1, Instituto de la Comunicación (InCom) de la Universidad Autónoma de Barcelona y Consejo del Audiovisual de Cataluña (CAC), Barcelona, p. 125-139.

Becerra, Martín (2003), Sociedad de la información: proyecto, convergencia, divergencia, Editorial Norma, Buenos Aires, 156 p.

Bolaño, César; G. Mastrini y F. Sierra (2005) (eds.), Economía política, comunicación y conocimiento. Una perspectiva crítica latinoamericana, La crujía, Buenos Aires, 429 p.

Bolaño, César y Alain Herscovici (2004), "Economia da informação e conhecimento: uma abordagem em termos de Economia Política", en Anais do VII Colóquio Brasil-França de Ciências da Comunicação e da Informção, mimeo, Porto Alegre.

Bolaño, César (2000), Industria cultural. Informação e capitalismo, Hucitec y Polis, São Pablo, 282 p.

Bolaño, César (1988), Mercado brasileiro de televisão, Editorial de la UFS, Aracajú, 175 p.

Bustamante, Enrique (coord.) (2003), Hacia un nuevo sistema mundial de comunicación. Las industrias culturales en la era digital, Gedisa, Barcelona, 379 p

Bustamante, Enrique (1999), La televisión económica. Financiación, estrategias y mercados, Gedisa, Barcelona, 220 p.

Capparelli, Sergio (1999), Enfim, sós: a nova televisão no cone sul, L\&PM, Porto Alegre, 181p.

Capparelli, Sergio y Venicio de Lima (2004), Televisão: desafios da pós-globalizaçao, Hacker editores, Sao Paulo, 162 p.

Carlsson, Ulla (2003), "The Rise and Fall of NWICO - and Then?: From a Vision of International Regulation to a Reality of Multilevel Governance", mimeo, ponencia presentada en el EURICOM Colloquium, Information Society: Visions and Governance, Venecia, Italia, Mayo de 2003.

Castells, Manuel y Peter Hall (1994), Las tecnópolis del mundo: la formación de los complejos industriales del siglo XXI, Alianza Editorial, Madrid, 363 p.

Castells, Manuel (1995), La ciudad informacional: tecnologías de la información, reestructuración económica y el proceso urbano-regional, Alianza Editorial, Madrid, 504 p.

Crovi Druetta, Delia (coord.) (2004), Sociedad de la Información y el conocimiento: entre lo falazy lo posible, La Crujía, Buenos Aires, 391 p.

Crovi Druetta, Delia y Cristina Girardo (2001), La convergencia tecnológica en los escenarios laborales de la juventud, UNAM, México. 
Crovi Druetta, Delia (coord.) (1995), Desarrollo de las industrias audiovisuales en México y Canadá, Facultad de Ciencias Políticas y Sociales, México DF, 277p.

Cruz Brittos, Valério y César Bolaño (comps.) (2005), Rede Globo: 40 anos de poder e hegemonia, Paulus, Sao Paulo, 373 p.

Del Valle Rojas, Carlos (2004), Metainvestigación de la comunicación en Chile, Universidad de la Frontera, Temuco, $130 \mathrm{p}$.

Fox, Elizabeth y Silvio Waisbord (eds.) (2002), Latin politics, global media, University of Texas Press, Austin, 204 p.

Fuenzalida, Valerio (2000), La televisión pública en América Latina. Reforma o privatización, Fondo de cultura económica, Santiago de Chile, 380 p.

García Canclini, Néstor (2004), Diferentes, desiguales y desconectados: mapas de la interculturalidad, Gedisa, Barcelona, 223 p.

García Canclini, Néstor y Carlos Moneta (coords.) (2004), Las industrias culturales en la integración latinoamericana, Eudeba, Buenos Aires, 342 p.

Getino, Octavio (1995), Las industrias culturales en la Argentina. Dimensión económica y políticas públicas, Colihue, Buenos Aires, 384p.

Getino, Octavio (2000), Las industrias culturales en el Mercosur: incidencia económica y sociocultural, intercambios y politicas de integración regional, Organización de Estados Americanos, Buenos Aires, $153 \mathrm{p}$.

Giordano, Eduardo y Carlos Zeller (1999), Políticas de televisión. La configuración del mercado audiovisual, Icaria, Barcelona, $230 \mathrm{p}$.

Guzmán Cárdenas, Carlos (2005), La dinámica de la cultura en Venezuela y su contribución al PIB, Convenio Andrés Bello, Caracas, 220 p.

Guzmán Cárdenas, Carlos (2003), Políticas y economía de la cultura en Venezuela, Ininco, Caracas, 192 p.

Herscovici, Alain (1994), Économie de la Culture et de la Communication, L'Harmattan, Paris.

Herscovici, Alain (2004), "Economia da Informação, Redes Eletrônicas e Regulação: Elementos de Análise", en Revista de Economia Política v. 24, n. n.1, São Paulo, p. 95-114.

Ianni, Octavio (1999), "La era del globalismo”, en Nueva Sociedad no 163, p. 92-106.

Jambeiro, Othon (2001), A tv no Brasil do século XX, Edufba, Salvador, 264 p.

Jambeiro, Othon (2000), Regulando a TV. Uma visão comparativa no Mercosul, Edufba, Salvador, 248p.

Katz, Claudio (1997), "El culturalismo en los estudios de tecnología”, en Causas y Azares no 6, Causas y Azares, Buenos Aires, p. 107-120.

Katz, Claudio (1998), "El enredo de las redes", en Voces y Culturas no 14, Voces y Culturas, Barcelona, p. 123-140.

Katz, Claudio (2001), Mito y realidad de la revolución informática, mimeo, en Portal EPTIC, Textos para Discussao II, www.eptic.he.com.br), consultado en marzo de 2002.

Marques de Melo, José y Luciano Sathler (orgs.), Direitos à cominicação na sociedade da informação, Universidade Metodista de San Pablo, San Pablo, 288 p. 
Mastrini, Guillermo y Martín Becerra (2006), Periodistas y magnates: estructura y concentración de las industrias culturales en América Latina, Prometeo, Buenos Aires, 330 p.

Mastrini, Guillermo (Ed.) (2005), Mucho ruido, pocas leyes. Economía y politicas de comunicación en la Argentina, La crujía, Buenos Aires, 320 p.

Mastrini, Guillermo y Diego de Charras (2004), Veinte años no es nada: del NOMIC a la CMSI, mimeo, ponencia al Congreso IAMCR 2004, Porto Alegre, Brasil. Disponible en www.catedras.fsoc.uba.ar/mastrini/investigaciones/mastrini_decharras.doc

Mastrini, Guillermo y César Bolaño (eds.) (1999), Globalización y monopolios en la comunicación en América Latina. Hacia una economía politica de la comunicación, Biblos, Buenos Aires, 250 p.

Miguel, Juan Carlos (1993), Los grupos multimedia. Estructura y estrategias en los medios europeos, Bosch, Barcelona, $348 \mathrm{p}$.

Muraro, Heriberto (1987), Invasión cultural, economía y comunicación, Legasa, Buenos Aires, 145 p.

Muraro, Heriberto (1974), Neocapitalismo y comunicación de masa, Eudeba, Buenos Aires, 244 p.

Murciano, Marcial (1992), Estructura y dinámica de la comunicación internacional, Bosch Barcelona, 252

p.

Postolski, Glenn, Ana Santucho y Daniel Rodríguez (2004), "Las alambradas mediáticas: concentración de la propiedad y sus consecuencias sobre el empleo en la prensa", mimeo, Observatorio Político, Social y Cultural de los Medios de la UTPBA, Buenos Aires, 16p.

Prado, Emili y Rosa Franquet (1998), "Convergencia digital en el paraíso tecnológico: claroscuros de una revolución”, en Zer n4, Facultad de Ciencias Sociales y de la Comunicación, Universidad del País Vasco, Bilbao, p. 15-40.

Quaderns del CAC (2005), XXV Aniversario del Informe MacBride. Comunicación internacional y politicas de comunicación, num. 21, CAC, Barcelona, $162 \mathrm{p}$.

Quirós, Fernando Y Francisco Sierra (dir.) (2001), Crítica de la economía política de la comunicación y la cultura, Comunicación Social ediciones, Sevilla, 276 p.

Reyes Mata, Fernando (1984), "El nuevo orden informativo reubicado: de la UNESCO a la UIT", en Comunicación y Cultura no. 11, Comunicación y Cultura, México, p. 9-16.

Rincón, Omar (comp.) (2001), Televisión pública: del consumidor al ciudadano, Convenio Andrés Bello, Bogotá, 316 p.

Schmucler, Héctor (1984), "Año mundial de la comunicación. Con penas y sin gloria", en Comunicación y Cultura no 11, Comunicación y Cultura, México DF, p. 3-8.

Sinclair, John (1999), Latin American television. A global view, Oxford University Press, Nueva York, $187 \mathrm{p}$.

Stolovich, Luis y otros (2002), La cultura es capital, Fin de siglo, Montevideo, 368 p.

Stolovich, Luis (2001), La lógica económica del empleo cultural, Inédito, Montevideo.

Stolovich, Luis, Graciela Lescano y José Mourelle (1997), La cultura da trabajo, Fin de Siglo, Montevideo.

Sunkel, Guillermo (coord.) (1999), El consumo cultural en América Latina: construcción teórica y líneas de investigación, Convenio Andrés Bello, Bogotá, 426 p. 
Sunkel, Guillermo y Esteban Geoffroy (2001), La concentración económica de los medios de comunicación, LOM, Santiago de Chile, 123 p.

Torres López, Juan, y Ramón Zallo (1991), "Economía de la información. Nuevas mercancías, nuevos objetos teóricos", en Telos n ${ }^{\circ}$ 28, Fundesco, Madrid, p. 54-67.

Vidal Beneyto, José (2002), La ventana global, Taurus, Madrid, 444p.

Yúdice, George (2002), El recurso de la cultura: usos de la cultura en la era global, Gedisa, Barcelona, $475 \mathrm{p}$.

Yúdice, George (2003), The expediency of cultura: uses of culture in the global era, Duke University Press, 466 p.

Zallo, Ramón (1988), Economía de la comunicación y la cultura, Akal, Madrid, 207 p.

Zallo, Ramón (1992), El mercado de la cultura (Estructura económica y politica de la comunicación), Tercera Prensa, Donostia, 245 p.

Zallo, Ramón (et al.) (1995), Industrias y politicas culturales en España y País Vasco, Servicio Editorial Universidad del País Vasco, Bilbao, 428 p.

Zallo, Ramón (2000), La crisis general de paradigmas. El caso de la economía y política de la comunicación y de la cultura, Mimeo, Bilbao, 14 p. 